\title{
ON CARDINALITY, COHOMOLOGY AND A CONJECTURE OF ROSENBERG AND ZELINSKY
}

\author{
BY \\ ROBERT E. MACRAE ( $\left.{ }^{1}\right)$
}

1. Introduction. It might be said that the cohomology of associative algebras (for an exposition see [1, Chapter IX]) first became of real interest when Hochschild showed [2, Theorem 4.1] that, for algebras of finite order over a field, the identical vanishing of the first cohomology group is equivalent to the classical notion of separability for such algebras. For commutative algebras of finite order over a field, this theorem had been shown some years earlier by E. Noether in the posthumous [4]. Then in 1956 Rosenberg and Zelinsky [5, Theorem 1] showed the surprising fact that if $S$ is an associative algebra over a field $K$ and the first cohomology group of $S$ vanishes identically, then $S$ is necessarily of finite order over $K$. They then went on to show that if $S$ is locally separable and of countable order over $K$, then the second cohomology group of $S$ vanishes identically [5, Theorem 4]. Zelinsky had already [6, p. 316] given an example to show that the countability hypothesis cannot be dropped. It was natural, therefore, for them to conjecture [5, bottom of p. 86] that the identical vanishing of the second cohomology group suffices to force countable order over the ground field-at least when $S$ is a field. By utilizing Kaplansky's remarkable piece of universal algebra [3], we are now able to give an affirmative answer to the conjecture of Rosenberg and Zelinsky-at least when $S$ is a field. For more general algebras the problem remains open.

I would like to thank H. Bass for a number of helpful conversations.

2. Results. If $S$ is a $K$-algebra, then $\operatorname{dim}_{K}(S)$ will denote the Hochschild dimension of $S$. If $S$ is a ring, then $\operatorname{gl} . \operatorname{dh}(S)$ will denote the global dimension of $S$. See [1] for further details.

LEMMA 2.1. Let $S$ be a commutative ring. If $\operatorname{gl} . \mathrm{dh}(S) \leqq 2$, then $S$ possesses no nontrivial nilpotent elements.

Proof. Let $N$ be the ideal of $S$ consisting of all the nilpotent elements of $S$. We wish to show $N=0$. Let $P$ be any maximal ideal of $S$ and denote the canonical extension of $N$ to the ring of quotients, $S_{P}$, by $N_{P}$. One knows that $N_{P}$ is the

Received by the editors January 7, 1964.

(1) This paper was partly written under grants NSF-GP-24 and NSF-GP-1904 from the National Science Foundation. 
ideal of nilpotent elements in $S_{P}$ and $N=0$ if and only if $N_{P}=0$ for all maximal ideals, $P$, in $S$. Furthermore, it is well known that $\operatorname{gl} . \operatorname{dh}\left(S_{P}\right) \leqq \operatorname{gl} . \operatorname{dh}(S)$. It suffices, therefore, to assume that $S$ has precisely one maximal ideal. In this case we will show the stronger fact that $S$ possesses no divisors of zero. Indeed, let $\mathscr{A}$ be a zero divisor and let $A$ be its annihilator. One has the following standard exact sequences:

$$
\begin{aligned}
& 0 \rightarrow A \rightarrow S \rightarrow \mathscr{A} S \rightarrow 0, \\
& 0 \rightarrow \mathscr{A} S \rightarrow S \rightarrow S / \mathscr{A} S \rightarrow 0 .
\end{aligned}
$$

Since gl.dh $(S) \leqq 2$, we see that $A$ is a projective $S$-module. Hence, by Kaplansky's result [3, Theorem 2], $A$ is free. Since it is an ideal in a commutative ring, $A$ must then be a principal ideal generated by a nondivisor of zero. This is, however, a contradiction since $\mathscr{A} A=0$.

COROLlaRY 2.2. Let $K$ be a field and let $S$ be a commutative, algebraic $K$ algebra. If $\operatorname{gl} . \mathrm{dh}(S) \leqq 2$, then $S$ is a von Neumann ring (i.e., $\mathscr{A} \in \mathscr{A}^{2} S$ for all $\mathscr{A} \in S)$.

Proof. Let $P$ be a prime ideal of $S$. One knows that $S_{P}$ is again algebraic over $K$. Hence the maximal ideal of $S_{P}$ consists only of nilpotent elements $\left(X^{n}=0\right.$ for some $n$ is the only possible equation over $K$ ). By Lemma 2.1, $S_{P}$ is a field. Let $\mathscr{A} \in S$ and let $S=S / \mathscr{A}^{2} S$. From the above, $\bar{S}_{P}^{-}$is a field for all prime ideals, $\bar{P}$ of $\bar{S}$. Hence $\bar{S}$ has no nilpotent elements aside from zero so $\mathscr{A} \in \mathscr{A}^{2} S$.

LEMMA 2.3. Let $K$ be a field and let $S$ be a commutative, algebraic $K$ algebra. If $\operatorname{dim}_{K}(S) \leqq 1$, then $\operatorname{dim}_{K}(S / A) \leqq 1$ for all proper ideals, $A$, of $S$.

Proof. By [1, Chapter IX, Proposition 7.4], $\operatorname{dim}_{K}\left(S \otimes_{K} S\right) \leqq \operatorname{dim}_{K}(S)+\operatorname{dim}_{K}(S)$. By [1, Chapter IX, Proposition 7.6], gl.dh $\left(S \otimes_{K} S\right) \leqq \operatorname{dim}_{K}\left(S \otimes_{K} S\right)$. Hence we have $\operatorname{gl} . \operatorname{dh}\left(S \otimes_{K} S\right) \leqq 2$. By Corollary 2.2, $S \otimes_{K} S$ is a von Neumann ring. By [3, Theorem 4], any projective ideal of $S \otimes_{K} S$ is generated by orthogonal idempotents. Consider the standard exact sequence

$$
0 \rightarrow J \rightarrow S \otimes_{K} S \rightarrow S \rightarrow 0 .
$$

Since $\operatorname{dim}_{K}(S) \leqq 1$, we see that $J$ is $\left(S \otimes_{K} S\right)$-projective and so is generated by orthogonal idempotents. Let $A$ be any proper ideal of $S$, and let $S=S / A$. We observe the following commutative, exact diagram:

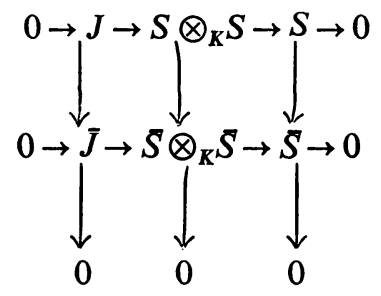


Since $J$ is generated by orthogonal idempotents so is $\bar{J}$ and thus $\bar{J}$ is $\left(S \otimes_{K} S\right)$ projective. In other words, $\operatorname{dim}_{K}(\bar{S}) \leqq 1$.

Our main result is now within reach.

THeOREM 2.4. Let $K$ be a field and let $L$ be an extension field. Then $\operatorname{dim}_{K}(L)$ $=1$ if and only if (i) $L$ is of countable (but not finite) order over $K$ and (ii) $L$ is a separable algebraic extension of $K$, or $L$ is a finite, separable extension of an intermediate field, $K(\mathscr{A})$, generated by a single transcendental element.

Proof. By the results of Rosenberg and Zelinsky [5] it suffices to show that if $\operatorname{dim}_{K}(L)=1$ and $L$ is algebraic over $K$ then $L$ is separable over $K$ and of countable but not finite order. We show first that $L$ is a separable extension of $K$. Let $\mathscr{A} \in L$. It is well known that $\mathscr{A}$ is separable if and only if $K[\mathscr{A}] \otimes_{K} K[\mathscr{A}]$ contains no nilpotent elements. Since $K$ is a field the injection,

$$
K[\mathscr{A}] \otimes_{K} K[\mathscr{A}] \rightarrow L \otimes_{K} L
$$

is a monomorphism. Hence it suffices to show that $L \otimes_{K} L$ has no nilpotent elements. By $\left[1\right.$, Chapter IX, Proposition 7.1], $\operatorname{dim}_{L}\left(L \otimes_{K} L\right)=\operatorname{dim}_{K}(L)$. By $[1$, Chapter IX, Proposition 7.6], gl.dh $\left(L \otimes_{K} L\right) \leqq \operatorname{dim}_{L}\left(L \otimes_{K} L\right)$. Hence gl.dh $\left(L \otimes_{K} L\right)$ $\leqq 1$. By Lemma $2.1, L \otimes_{K} L$ has no nilpotent elements. In order to show that the order of $L$ over $K$ is countable we first note that the classical Hochschild Theorem [1, Chapter IX, Theorem 7.10] assures us that the order cannot be finite. Consider now the standard exact sequence

$$
0 \rightarrow J \rightarrow L \otimes_{K} L \rightarrow L \rightarrow 0 .
$$

We have already shown above that $\operatorname{gl} . \operatorname{dh}\left(L \otimes_{K} L\right) \leqq 1$. Hence by Corollary 2.2 and [3, Theorem 4], $J$ is generated by orthogonal idempotents. We claim first that these idempotent generators form a countable set. Indeed, let $M$ be the algebraic closure of $K$ and consider the following exact sequences

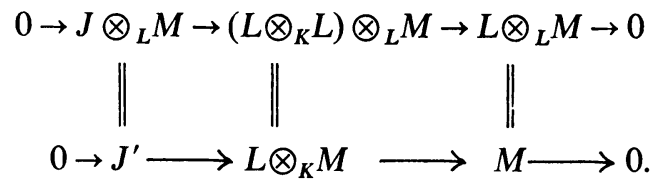

One observes that $J^{\prime}$ is a maximal ideal of $L \otimes_{K} M$ generated by orthogonal idempotents. Furthermore, by [1, Chapter IX, Proposition 7.1], $\operatorname{dim}_{M}\left(L \otimes_{K} M\right)$ $=\operatorname{dim}_{K} L=1$. Let $S=L \otimes_{K} M$, let $u_{1}, \cdots$ be orthogonal idempotents generating the maximal ideal $J^{\prime}$ and let $A_{1}, \cdots$ be ideals of $S$ maximal with respect to the two properties (i) $A_{i} \leqq u_{i} S$ and (ii) $u_{i} \notin A_{i}$. Let $A=\sum A_{i}$. By Lemma 2.3, $\operatorname{dim}_{M}(S / A)$ $\leqq 1$. One observes that the canonical images of $u_{1}, \cdots$ in $S / A$ are orthogonal, primitive idempotents generating a maximal ideal of $S / A$. Since $M$ is algebraically 
closed, $S / A$ is isomorphic to a direct sum of copies of $M$ (one for each $u_{i}$ ) with an identity element adjoined. It is, however, known [5, last paragraph of p. 86] that, for such an algebra, $\operatorname{dim}_{M}(S / A) \leqq 1$ implies that the set of generating idempotents is, at most, countable. Since the cardinality of the set of $u_{1}, \cdots$ is clearly the same as that of the set of orthogonal idempotents generating $J$, we have made good our claim. It now remains only to prove that $L$ possesses a countable $K$ basis. Let $s_{1}, \cdots$ be a $K$-basis for $L$ and let $v_{1}, \cdots$ be a (necessarily countable) set of orthogonal idempotents generating $J$. We have the equations

$$
v_{i}=\sum \lambda_{i j k} s_{j} \otimes s_{k},
$$

where the coefficients are in $K$ and each sum is finite. The subset of the $s_{1}, \cdots$ involved in the expression of the $v_{1}, \cdots$ is clearly countable. Let $N$ be the intermediate field generated by this subset. Clearly $N$ is of countable order over $K$. Consider the exact commutative diagram:

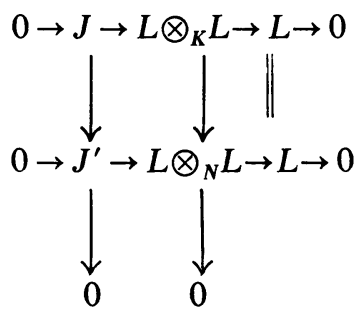

It is clear from our construction that $J^{\prime}=0$. But this can only happen when $N=L$.

\section{REFERENCES}

1. H. Cartan and S. Eilenberg, Homological algebra, Princeton Univ. Press, Princeton, ZJJ., 1956.

2. G. Hochschild, On the cohomology groups of an associative algebra, Ann. of Math. (2) 46 (1945), 58-67.

3. I. Kaplansky, Projective modules, Ann. of Math. (2) 68 (1958), 372-377.

4. E. Noether, Idealdifferentiation und Differente, J. Reine Angew. Math. 188 (1950), 1-21.

5. A. Rosenberg and D. Zelinsky, Cohomology of infinite algebras, Trans. Amer. Math. Soc. 82 (1956), 85-98.

6. D. Zelinsky, Raising idempotents, Duke Math. J. 21 (1954), 315-322.

COLUMBia University

New York, New York 